Rev. Elet. em Gestão, Educação e Tecnologia Ambiental (e-ISSN: 2236-1170)

\title{
UTILIZAÇÃO DE FUNGO DE BAMBU NA BIORREMEDIAÇÃO DE SOLO CONTAMINADO
}

\author{
Mayara Barbosa Silva', Josimara Nolasco Rondon² \\ 1'Graduação em andamento em Ciências Biológicas na Universidade Católica Dom Bosco. may 153130@hotmail.com \\ ${ }^{2}$ Graduação em Ciencias Biologicas pela Universidade Federal do Mato Grosso do Sul, Mestrado em Biologia Vegetal \\ pela Universidade Estadual de Campinas (2001) e Doutorado em Biologia Vegetal pela Universidade Estadual de \\ Campinas (2006) ; Atualmente é professora visitante e pesquisadora da Universidade Católica Dom Bosco.
} 4132@ucdb.br

http://dx.doi.org/10.5902/223611707757

\section{RESUMO}

A biorremediação é uma tecnologia para tratar locais contaminados mediante o uso de agentes biológicos capazes de modificar ou decompor poluentes. Considerando que todas as plantas podem ser hospedeiras de um ou mais fungos endófitos, e esses apresentam grande potencial biotecnológico o trabalho teve como objetivo estudar a viabilidade de utilização de fungo endófitico de Bambusa vulgaris (Poaceae) no biotratamento de solos contaminados. Para isso foram feitos contaminações ex situ de solos com óleo diesel, chorume e o herbicida Roundup e o fungo na diluição de $5 \times 10^{5}$ conídios $/ \mathrm{mL}$ e semanalmente foram avaliados o tamanho de colônias para crescimento fúngico, o calculo do volume inicial e final do poluentes testados pelo teste de Tukey a $5 \%$ de probabilidade. O fungo endófitico Fusarium moliniforme Sheldon teve $99 \%$ de sobrevivência nos três contaminantes avaliados no período de 30 dias. 0 chorume apresentou maior consumo e crescimento do fungo. As amostras da contaminação com Roundup que tiveram acréscimo de solução nutritiva obteve maior crescimento do que as que não foram acrescidas da solução evidenciando a importância do incremento de nutrientes para a bioestimulação para alguns tratamentos. Maiores quantidades dos contaminantes apresentam melhores resultados no processo de tratamento de áreas contaminadas.

Palavras-chave: bioestimulação, crescimento, Fusarium moliniforme, degradação, contaminantes

\begin{abstract}
Bioremediation is a technology for treating contaminated sites by the use of biological agents capable of modifying or decomposing pollutants. Whereas all plants can host one or more fungal endophytes, and these have great biotechnological potential the work aimed to study the feasibility of using endophytic fungi from Bambusa vulgaris (Poaceae) in the bioremediation of contaminated soils. For that were made soil contamination ex situ with diesel oil, chorume and the herbicide Roundup and fungus in the dilution of $5 \times 105$ conidios / $\mathrm{mL}$ were evaluated weekly the size of colonies and the fungal growth, the calculation of the initial and final volume of the pollutants tested by Tukey test at $5 \%$ probability. The endophytic fungus Fusarium moliniforme Sheld had $99 \%$ survival in the three contaminants evaluated within 30 days. The chorume had
\end{abstract}


Rev. Elet. em Gestão, Educação e Tecnologia Ambiental (e-ISSN: 2236-1170)

higher consumption and growth fungus. The samples that were contaminated with Roundup addition of nutrient solution obtained higher growth than that of the solution were added indicating the importance of nutrients for growth of biostimulation for some treatments. Larger quantities of the contaminants present better results in the treatment process of contaminated areas.

Key words: biostimulation, growth, Fusarium moliniforme, degradation, contaminants

\section{INTRODUÇÃO}

A biorremediação é uma nova tecnologia para tratar locais contaminados mediante o uso de agentes biológicos capazes de modificar ou decompor poluentes alvos. Consistindo no principio da remoção de poluentes do ambiente natural e/ou a sua conversão em produtos menos prejudiciais, utilizando, para isso, comunidades de microrganismos endógenos do ambiente contaminados (MARIANO, 2006).

O objetivo da biorremediação no tratamento em áreas contaminadas é induzir ou acelerar os processos biológicos naturais de reciclagem de compostos de interesse, incluindo compostos orgânicos ou inorgânicos utilizando a capacidade intrínseca dos microorganismos de degradar matéria orgânica para degradar compostos orgânicos tóxicos, tanto de origem natural como compostos sintéticos (DIAS, 2000).

A biorremediação varia de acordo com o tipo de tratamento, as técnicas são denominadas in situ e ex situ, adotadas considerando não só os poluentes, mas o custo dos processos e, principalmente, a concentração final do contaminante, no término do tratamento, como aceitável para o tipo de resíduo e para o uso futuro da área (OLIVEIRA, 2008).

Sabendo que os fungos são microorganismos produtores de um sistema enzimático complexo, capazes de degradar substâncias químicas de estrutura complexa, de maneira a produzir moléculas mais simples e mais facilmente assimiláveis. Em função desses mecanismos de degradação, a utilização de fungos é uma alternativa biológica viável para a remediação de solos contaminados (BRITO, 2004).

A remoção dos poluentes por fungos começou a ser estudado nos últimos trinta anos do século XX (SOARES et al., 2011). Os primeiros trabalhos com a biorremediação através de fungos foi com o uso de Saccharomyces cerevisiae para remoção de lindano e dieldrin por Nobles (1975) seguido pelo estudo de Khindaria et al., (1975) que estudaram a degradação de heptacloro por fungos do gênero Aspergillus.

Os compostos que exigem maior preocupação ambiental são o benzeno, tolueno, etilbenzeno e xilenos (isômeros: orto-, meta- e para-xileno). Esses compostos são conhecidos como BTEX, sendo definidos como hidrocarbonetos monoaromáticos, cujas estruturas moleculares possuem como característica principal a presença do anel benzênico. São usados, principalmente, herbicidas, em solventes e em combustíveis e são os constituintes mais solúveis na fração da gasolina (ANDRADE et al., 2010). Esses compostos aromáticos são tóxicos não só ao solo, mas também ao ser humano atuando como depressores do sistema nervoso central e apresentam toxicidade crônica mesmo em concentrações da ordem muito pequenas (WATTS et al., 2000). 
Rev. Elet. em Gestão, Educação e Tecnologia Ambiental (e-ISSN: 2236-1170)

E os fungos participam nas reações de transformação assimilando hidrocarbonetos como única fonte de carbono e energia, resultando na formação de dióxido de carbono. Essa transformação pode ser também através da reação de oxi-redução. A oxidação de hidrocarbonetos aromáticos, por exemplo, iniciado com uma oxidação de óxidos de areno pelo citocromo (SINGH, 2006).

Algumas avaliações feitas por Mazzeo (2009) de genotoxicidade e mutagenicidade, realizadas com células meristemáticas e $\mathrm{F} 1 \mathrm{de} A$. cepa, indicaram que o processo de biodegradação por fungos foi eficiente na biodegradação da mistura aquosa do BTEX, por diminuir os efeitos genotóxico e mutagênico, indicando que os compostos da mistura foram degradados em metabólitos não tóxicos para as células.

Estudos feitos por Santos e Linardi (2004) utilizaram fungos dos gêneros Aspergillus, Geotrichum, Fusarium, Penicillium e Phanerochaete, entre outros, para biodegradação de compostos fenólicos, pois são capazes de degradar compostos aromáticos em seu metabolismo por meio de enzimas catabólicas celulases, lacases, proteases e fenol-hidroxilases.

Os microrganismos endófitos são aqueles que habitam o interior das plantas, sendo encontrados em órgãos e tecidos vegetais como as folhas, ramos e raízes. Esta comunidade endofítica é constituída principalmente por fungos e bactérias, e ao contrário dos microrganismos patogênicos, não causam prejuízos aos seus hospedeiros. Muito pelo contrário, os endófitos podem desempenhar relevante função para a sanidade vegetal, já que atuam como agentes controladores de microrganismos fitopatogênicos, além de poder funcionar também no controle de insetos e até proteger (NETO et al., 2003).

Considerar se que todas as plantas podem ser hospedeiras de um ou mais fungos endófitos, já que estes foram encontrados em todas as espécies de plantas pesquisadas até o momento, podendo haver ou não interespecificidade e/ou dependência. O conhecimento destes microrganismos, de sua biodiversidade, da filogenia e das relações ecológicas bióticas e abióticas, é promissor e de suma importância para se entender as diferentes interações e funcionalidades em um ecossistema, para a bioprospeç̧ão e para a biotecnologia (RODRIGUES, 2010).

O primeiro estudo sobre a capacidade de degradação de poliuretano (PUR) por fungos endófiticos define as promissoras fontes de diversidade desses microrganismos quando se trata de atividades importantes para biorremediação (RUSSEL et al., 2011).

O presente trabalho objetivou investigar se o fungo endófitico de Bambusa vulgaris (Poaceae) promoveria um efeito positivo no biotratamento de solos contaminados com chorume, óleo diesel e Roundap.

\section{METODOLOGIA}

Das estacas de Bambusa vulgaris (Poaceae) foi isolado o fungo em placas de petri contendo o meio Potato Dextrose ágar (PDA), identificado por meio de microscopia óptica como Fusarium moliniforme pela literatura consutada (The Fusarium Laboratory Manual de Leslie e Summerell (2006). Penicillium niger foi usado como comparação na análise microbiológica de colonias crescidas em PDA. Foram usadas $25 \mathrm{~g}$ de areia lavada para frascos de vidro de $100 \mathrm{~mL}$. Um outro experimento utilizou-se, $50 \mathrm{~mL}$ contendo meio de cultivo de Mangifera indica L. (manga) com óleo 
Rev. Elet. em Gestão, Educação e Tecnologia Ambiental (e-ISSN: 2236-1170)

diesel. Em seguida. os frascos, areia lavada, o meio de cultivo de manga e o óleo diesel e as estacas foram autoclavados separadamente, por 30 minutos a $121^{\circ} \mathrm{C}$ a $1 \mathrm{~atm}$. Através dos isolados fúngicos foram inoculados em cada pote de vidro com areia, uma diluição de $5 \times 10^{5}$ conídios $/ \mathrm{mL}$ em todos os tratamentos com exceção dos tratamentos testemunhas, nesses foram colocados as próprias estacas de bambu trituradas autoclavadas. A sobrevivência de Fusarium moliniforme foi observada nos experimentos testes por analises de sobrevivência/resistência do aos poluentes (chorume, herbicida Roundup e óleo diesel) por 30 dias. Realizou-se o Teste i: Sobrevivência do fungo ao chorume; o Teste ii: Sobrevivência do fungo ao óleo diesel; e o Teste iii: Sobrevivência do fungo ao herbicida Roundup.

Para avaliação do crescimento fúngico foram realizadas semanalmente as avaliações do número de colônias, crescimento e declínio das colônias, e análises semanais da dimensão das colônias de Fusarium moliniforme das amostras de solo contaminado com $12 \mathrm{~mL}$ de Roundup e 12 $\mathrm{mL}$ de chorume. Realizou-se análise do tamanho das colônias de dez amostras de solo contaminados com roundup e água destilada e de dez amostras de solo contaminadas com roundup e solução nutritiva. Nos experimentos de consumo dos poluentes foram avaliados a descontaminação do substrato conhecendo o volume inicial e final do poluente de casa amostra, pesados em balança de precisão. Foi realizado análise do consumo (em $\mathrm{mL}$ ) dos três contaminantes pelo fungo sobre as mesmas condições. A dimensão das colônias em relação à quantidade do poluente também foram verificadas em dez amostras com $5 \mathrm{~mL}$ de chorume e dez amostras com $8 \mathrm{~mL}$ de chorume.

\section{RESULTADOS E DISCUSSÃO}

O fungo Fusarium moliniforme apresentou sobrevivência em todos os contaminantes (figura 1), apresentando crescimento nos trinta dias de avaliação.
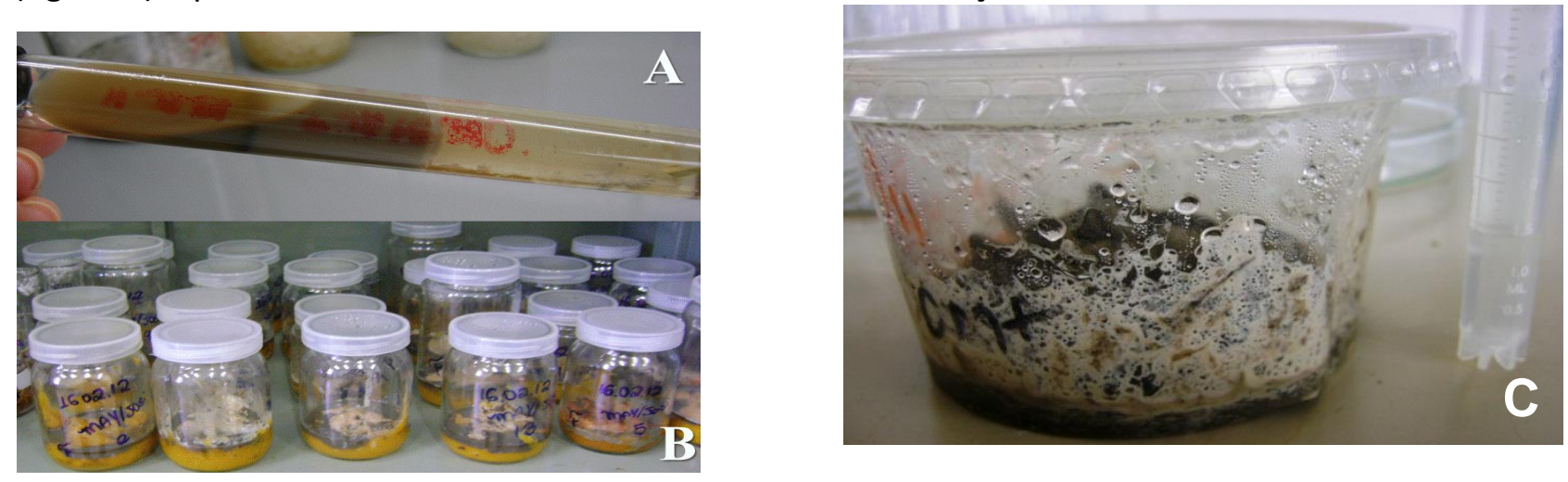

Figura 1. Crescimento e sobrevivência do fungo ao óleo diesel. A- Estacas pulverizadas de bambu misturadas ao óleo diesel. B- Frascos de vidro contendo meio de Mangifera indica L. e óleo diesel.

C- Estacas de bambu trituradas e misturadas com óleo diesel e plástico PVC. Ao lado, um eppendorf de $5 \mathrm{~mL}$, após biotransformação do óleo diesel em água.

No teste de sobrevivência foi simulado um ambiente contendo óleo diesel, plástico PVC e diferentes tipos de substrato e estacas de Bambusa vulgaris com presença visível do fungo 
Rev. Elet. em Gestão, Educação e Tecnologia Ambiental (e-ISSN: 2236-1170)

Fusarium moliniforme (Figura 1). Após 15 dias da contaminação foi percebida a "transpiração" da amostra. Feitas coletadas diárias dessa água em 14 dias chegando a um total de $60,7 \mathrm{~mL}$ que foi realizado teste microbiológico para análise da potabilidade pelo método de contagem em placa. Testes em duplicata com o meio MacConey para deteç̧ão de bactérias gram negativas, meio bacteriológico e no meio Potato dextrose ágar (PDA) para detecção de fungos (tabela 1).

Tabela 1. Análise microbiológica da água pelo método de contagem em placa UFC/mL.

\begin{tabular}{cccc}
\hline Meio & 10 ? l & $0,1 \mathrm{~mL}$ & $1 \mathrm{~mL}$ \\
\hline Mac Coney & $0,1 \mathrm{UFC} / \mathrm{ml}$ & $1 \mathrm{UFC} / \mathrm{ml}$ & $10 \mathrm{UFC} / \mathrm{ml}$ \\
bacteriológico & $9 \mathrm{UFC} / \mathrm{ml}$ & $90 \mathrm{UFC} / \mathrm{ml}$ & $900 \mathrm{UFC} / \mathrm{ml}$ \\
$\begin{array}{c}\text { PDA* } \\
\begin{array}{c}\text { Fusarium } \\
\text { moniliforme } \\
\text { Aspergillus niger }\end{array}\end{array}$ & $2,5 \mathrm{UFC} / \mathrm{ml}$ & $25 \mathrm{UFC} / \mathrm{ml}$ & $250 \mathrm{UFC} / \mathrm{ml}$ \\
& $1,5 \mathrm{UFC} / \mathrm{ml}$ & $15 \mathrm{UFC} / \mathrm{ml}$ & $250 \mathrm{UFC} / \mathrm{ml}$ \\
\hline
\end{tabular}

*No meio PDA as colônias foram calculados de acordo com a espécie do fungo.

Com a análise semanal dos tamanhos das colônias (tabela 2) foi possível a percepção satisfatória do crescimento do Fusarium moliniforme, sendo um bom indicador não só do consumo dos contaminantes, mas como também da eliminação de alguns compostos dos mesmos que servem de nutriente para seu crescimento.

Tabela 2. Médias das análises semanais do tamanho das colônias de Fusarium moliniforme em solo contaminado com $12 \mathrm{~mL}$ de Roundup e de chorume.

\begin{tabular}{cccc}
\hline Tempo (Dias) & Controle & Roundup & Chorume \\
\hline 7 & $1,14 \mathrm{~b}$ & $1,42 \mathrm{~b}$ & $1,84 \mathrm{a}$ \\
14 & $1,16 \mathrm{~b}$ & $1,48 \mathrm{~b}$ & $1,89 \mathrm{a}$ \\
21 & $1,28 \mathrm{~b}$ & $1,60 \mathrm{a}$ & $2,06 \mathrm{a}$
\end{tabular}

Médias seguidas por letras diferentes diferem estatisticamente pelo teste de Tukey no nível de $5 \%$ de probabilidade.

De acordo com a Tabela 2, observa-se que houve diferença significativa ao nível de $5 \%$ de probabilidade pelo teste Tukey, entre as médias do tamanho das colônias das amostras evidenciando o chorume como contaminante que teve maior crescimento fúngico. As amostras do tratamento 2 (figura 2) com acréscimo de solução nutritiva apresentou um aumento significante comparada com o as amostras de roundup com acréscimo de água destilada corroborando diversos estudos e apresentando a importância da bioestimulação inicial por acréscimos de nutrientes ao processo. 
SILVA \& RONDON, v(10), no 10, p. 2175-2184, JAN-ABR, 2013.

Rev. Elet. em Gestão, Educação e Tecnologia Ambiental (e-ISSN: 2236-1170)
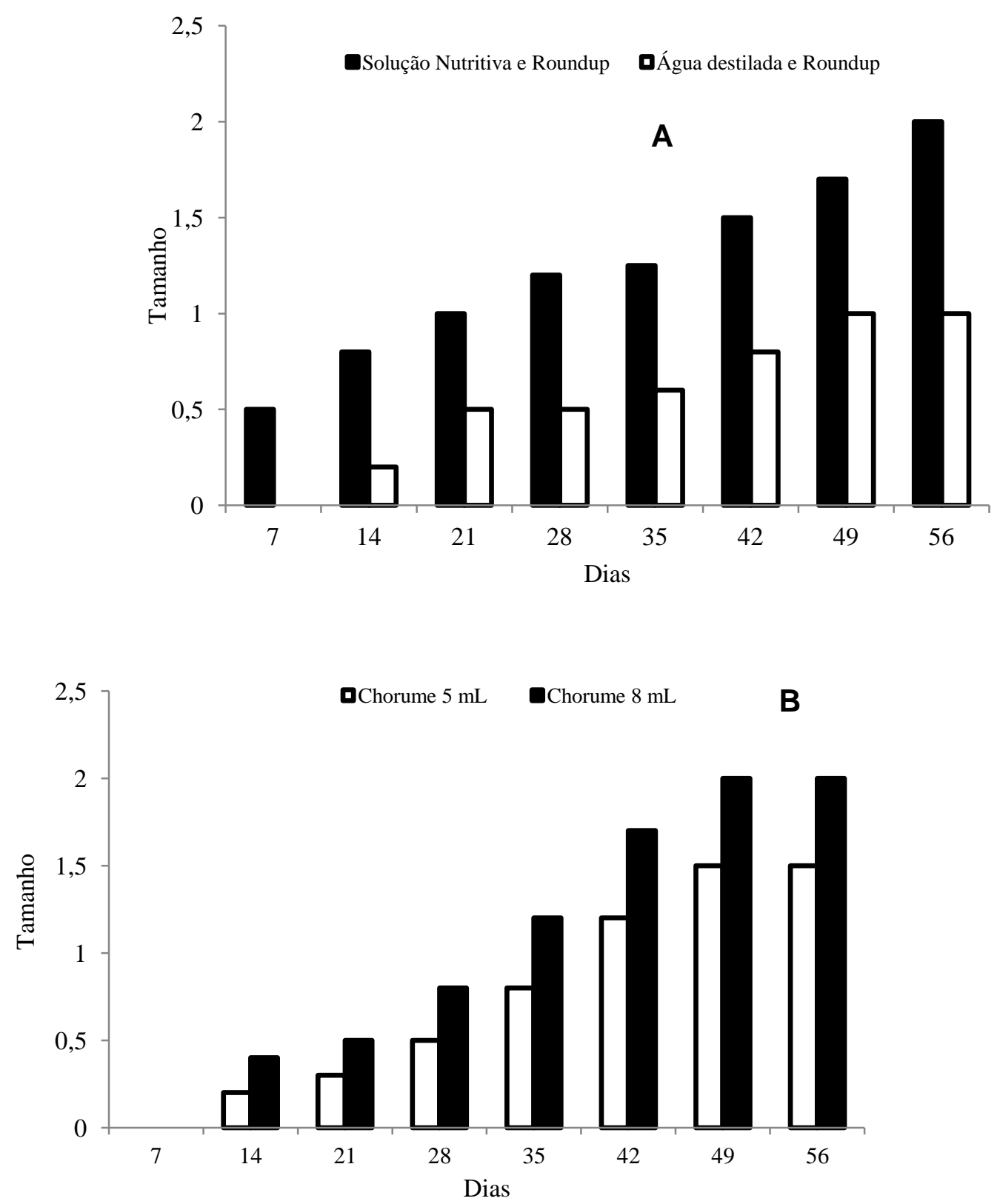
Rev. Elet. em Gestão, Educação e Tecnologia Ambiental (e-ISSN: 2236-1170)

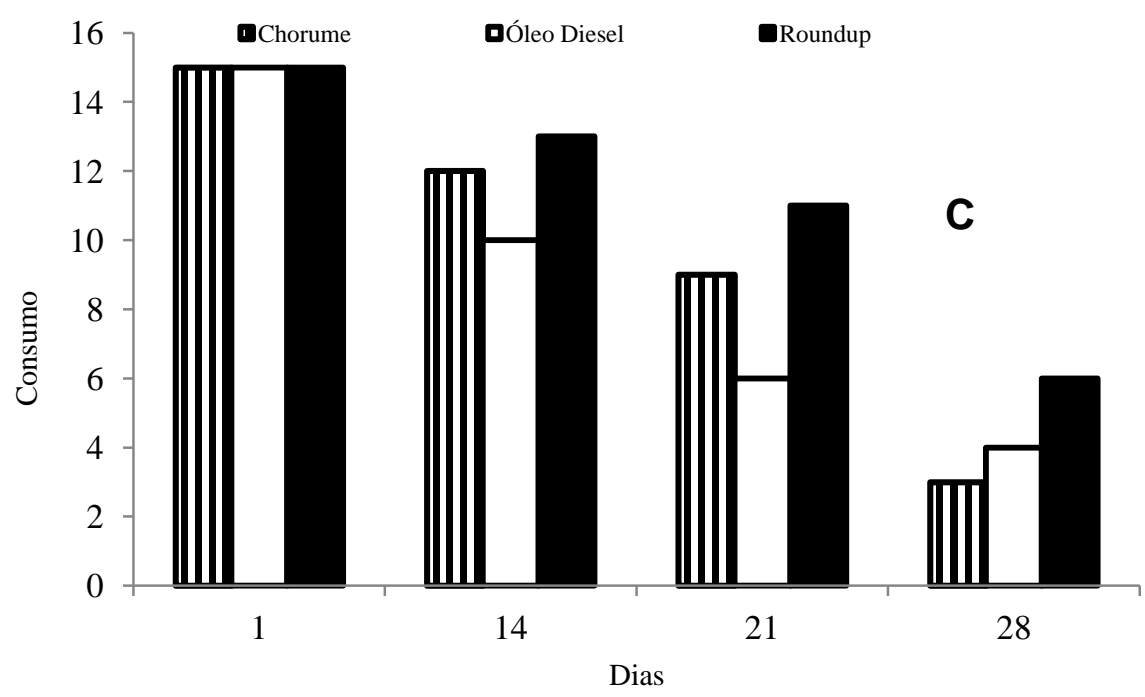

Figura 2. Análise do tamanho das colônias das amostras de solo contaminadas com roundup e água destilada e das amostras contaminadas com roundup e solução nutritiva (A) e chorume (B).

Análise do consumo em $\mathrm{mL}$ dos contaminantes pelo Fusarium moliniforme sobre as mesmas condições (C).

O que pode ser explicado pela necessidade de alguns poluentes precisarem da introdução de nutrientes e/ou surfactantes, como objetivo de aumentar a atividade microbiana já que os mesmos não conseguem suprir sozinhos as exigências dos microrganismos (GAYLARDE et al., 2005). E assim reduzindo substancialmente o tempo do biotratamento (BENTO et al., 2005).

A descontaminação foi evidenciada pelo consumo em $\mathrm{mL}$, ou seja, pela diminuição do volume inicial do final (Figura 3), mas também foi verificado visualmente o clareamento gradual dos contaminantes tanto o chorume, como o roundup (figura $3 \mathrm{~A}, \mathrm{~B}, \mathrm{C}$ e D), como o óleo diesel (figura 1A). A mudança de cor dos contaminantes tem sido relatada em diversos estudos (Martinez et al., 2008; Clemente et al., 2001; Mariano et al., 2006) e também no estudo de Cookson (1995) que a passagem da cor preta característica do óleo diesel para a cor cinza serve como indicador de remediação de alguns compostos tóxicos. 


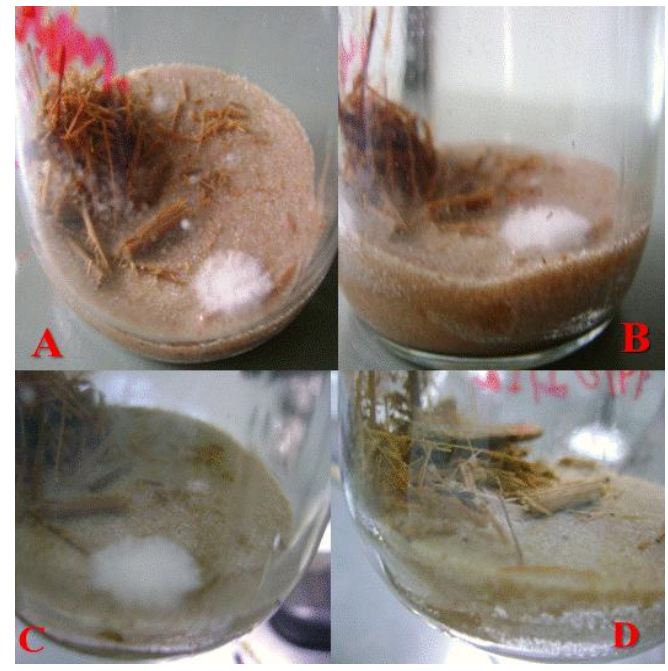

Figura 3. Amostras contaminadas com $10 \mathrm{ml}$ do herbicida roundup. A-B-C. Solução nutritiva mais roundup; D. Amostra de roundup com água destilada.

E também o clareamento como neutralização dos compostos de alguns herbicidas nos estudos de Vitali et al. (2006).O poluente que teve maior consumo foi o chorume o que é explicado pela sua fácil assimilação pelos microrganismos devido a uma importante fração orgânica encontrada no chorume que é constituída por proteínas, carboidratos e lipídios que e de suma importância para o crescimento mais acelerado nesse contaminante (Lema et al.,1988). Obteve maior crescimento (tamanho de colônia) as amostras de chorume que obtiveram maior quantidade do poluente, pois, quando as concentrações dos poluentes são baixas, de acordo com Bento et al. (2005) os microrganismos podem não obter bom crescimento e produzir as enzimas necessárias para o biotratamento das áreas contaminadas. Sendo uma afirmação otimista pra o tratamento de grandes áreas contaminadas com esse poluente.

O solo é um ambiente heterogêneo é possível que os ensaios com inóculos fúngicos apresentem diferentes graus de sucesso (BAMFORTH \& SINGLETON, 2005). Mas acelerar o crescimento ou aumentar a quantidade de fungos exercem efeitos benéficos, diminuindo a quantidade de poluentes tóxicos disponíveis, desde que sejam dadas as condições adequadas, especialmente para os poluentes difíceis de serem degradados (SINGH, 2006).

\section{CONCLUSÕES}

Fusarium moliniforme é um bom indicador para consumo de contaminantes, atuando na eliminação de roundap, chorume e óleo diesel. Pode ser usado no biotratamento de solos contaminados. 


\section{REFERÊNCIAS}

ANDRADE, J.A.; AUGUSTO, F.; JARDIM, I.C.S.F. Biorremediação de solos contaminados por petróleo e seus derivados. Eclética Química, São Paulo, v. 35, n. 3, p. 17-43, 2010.

BAMFORTH, S.; SINGLETON, I. Bioremediation of polycyclic aromatic hydrocarbons: current knowledge and future directions. Journal of Chemical Technology and Biotechnology, v. 80, n.7, p.723-736, 2005.

BENTO, F.M.; CAMARGO, F.A.O.; OKEKE. B. C.; FRANKENBERGER, W. Comparative bioremediation of soils contaminated with diesel oil by natural attenuation, biostimulation and bioaugmentation. Bioresource Technology, $v$. 96, 1049-1055, 2005.

BRITO, N. N.; ZAMORA, P. P.; NETO, A. L. O.; DE BATTISTI, A.; PATERNIANI, J. E. S.; PELEGRINI, R. T. Utilização de fungos na remediação de efluentes industriais. IV Fórum de Estudos Contábeis, Faculdades Integradas Claretianas, Rio Claro, SP, 2004.

CLEMENTE, A.R.; TANIA, A.; ANAZAWA, T.A.; DURRANT, L.R. biodegradation of polycyclic aromatic hydrocarbons by soil fungi. Brazilian Journal of Microbiology, 2001.

COOKSON, L. J. Reliability of poly B-411, a polymeric anthraquinone based dye, in determining the rot type caused by wood-inhabiting fungi. Applied and Environmental Microbiology, v.61, n. 2, 801-803, 1995.

DIAS, A. E. X. O. Biorremediação de áreas afetadas por resíduos sólidos tóxicos. In: SISINNO, C. L. S.; OLIVEIRA, R. M. (Org.) Resíduos Sólidos, Ambiente e Saúde: uma visão multidisciplinar. Rio de Janeiro: Editora Fiocruz, 142 p, 2000.

GAYLARDE, C. C.; BELLINASO, M. D. L.; MANFIO, G. P. Aspectos biológicos e técnicos da biorremediação de xenobióticos. Biotecnologia Ciência \& Desenvolvimento, n.34, 2005.

KHINDARIA, A.; GROVER, T.A.; AUST, S.D. Reductive dehalogenation of aliphatic halocarbons by lignin peroxidase of Phanerochaete chrysosporium. Environmental Science Technology, v. 29, p.719-725, 1975.

LEMA, J. M., et al. Characteristics of landifill leachates and alternatives for their treatment: a review. Water, Air \& Soil Pollution, v. 40, 223-250, 1988.

LESLIE, J. F.; SUMMERELL, B. A. The Fusarium Laboratory Manual. Blackwell Publishing, 2006.

MARTINEZ, C.O.; SILVA, C.M.M.S.; FAY, E. F. Caracterização de Bactérias e Fungos envolvidos na Degradação de Sulfentrazona em Solos. Jaguariúna: Embrapa Meio Ambiente. 22 p. - (Embrapa Meio Ambiente. Boletim de Pesquisa e Desenvolvimento, 2008.

MARIANO, A. P. et al. Laboratory study on the bioremediation of diesel oil contaminated soil From a petrol station. Brazilian Journal of Microbiology, 2007.

MARIANO, A. P. Avaliação do potencial de biorremediação de solos e de águas subterrâneas contaminados com óleo diesel. Tese (doutorado) - Universidade Estadual Paulista, Instituto de Geociências e Ciências Exatas, Rio Claro: [s.n.], 2006.

MAZZEO, D. E. C. Avaliação dos efeitos citotóxicos, Genotóxicos e mutagênicos do btex, antes e após o processo de biorremediação por microrganismos, utilizando os sistemas teste de Allium cepa e cultura de células de mamífero. Dissertação (mestrado) - Universidade Estadual Paulista, Instituto de Biociências de Rio Claro, Rio Claro : [s.n.], 2009. NETO, P. A. D. S. P.; AZEVEDO, J. L.; ARAUJO, W. L. Microrganismos Endofíticos. Biotecnologia Ciência \& Desenvolvimento, n. 29, 2003. 
NOBLES, M.K. Identification of cultures of wood-inhabiting. Hymenomycetes. Canadian Journal of Botany, v. 43, p. 1097-1139, 1975.

OLIVEIRA, S. D. Avaliação das técnicas de bioaumento fúngico e bioestímulo em processos de biorremediação utilizando solo contaminado por petróleo. (Dissertação) - Universidade Federal do Rio de Janeiro, Escola de Química, 2008.

RODRIGUES, R.L. Fungos endofíticos associados a Vellozia compacta Mart. ex Schult. F. (Velloziaceae) presente em afloramentos rochosos nos estados de Minas Gerais e Tocantins. Dissertação Universidade Federal de Ouro Preto do Programa de Pós-Graduação em Ecologia de Biomas Tropicais. Ouro Preto - MG, 2010.

RUSSELL, J.R., et al. Biodegradation of Polyester Polyurethane by Endophytic Fungi. Applied and Environmental Microbiology, doi:10.1128/AEM.00521-11 AEM Accepts, published online ahead of print, 2011.

SANTOS, V.L.; LINARDI, V.R. Biodegradation of phenol by a filamentous fungi isolated from industrial effluents identification and degradation potential. Process Biochemistry, v. 39, n. 8, p. 1001-1006, 2004.

SINGH, H. Mycoremediation: fungal bioremediation. John Wiley \& Sons, Inc. Hoboken, New Jersey.617p. 2006.

SOARES, L. A.; et al. Fungos na biorremediação de áreas degradadas. Arquivos do Instituto Biológico, São Paulo, v. 78, n. 2, p.341-350, 2011.

VITALI, V. M. V., et al. Screening mitosporic fungi for organochlorides degradation. Brazilian Journal of Microbiology, 2006.

WATTS, R.J.; HALLER, D.R.; JONES, A.P.; TEEL, A.L. A foundation for the risk-based treatment of soline contaminated soils using modified Fenton's reactions. Journal Hazard. Mater, v. 76, p.73-89, 2000. 